\title{
Detection of Genetic Components of Variation for Yield, Fibre and Quality Traits in Flax (Linum usitatissimum L.)
}

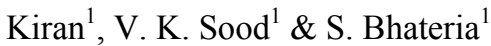 \\ ${ }^{1}$ Dept. of Crop Improvement, CSK Himachal Pradesh Krishi Vishvavidyalaya, Palampur, India \\ Correspondence: Kiran, Dept. of Crop Improvement, CSK Himachal Pradesh Krishi Vishvavidyalaya, Palampur, \\ 176062 (H. P.), India. Tel: 91-1894-230-391. E-mail: angelkiran@hotmail.com
}

Received: May 4, 2012 Accepted: May 22, 2012 Online Published: August 31, 2012

doi:10.5539/jas.v4n10p224 URL: http://dx.doi.org/10.5539/jas.v4n10p224

\begin{abstract}
The research was carried out using generation mean analysis derived from crosses of TL-11 x Him Alsi-2, Turkey x Binwa and TL-43 x Binwa at two locations i.e. E1 and E2. Out of the three crosses, TL $-11 \times$ Him Alsi-2 was the best cross in both the locations. This cross invariably has given 2-3 times higher yield than the remaining crosses. Therefore, the results obtained in the present investigation with respect to the above best cross for generation mean analysis suggested that both additive and dominance gene effects were important for most of the traits but dominance was predominant as compared to additive effects. Duplicate type of gene action was observed in the expression of days to 50 per cent flowering, seed yield per plant, plant height, technical height and fibre traits in E2 for the above cross which implies the use of biparental approach and selection to be deferred to later generations. Complimentary type of gene action was observed for biological yield per plant, seed yield per plant which implies the use of biparental approach and early generation selection. Therefore, it appears worthwhile to go for biparental matings or few cycles of recurrent selection for the accumulation of favourable genes for seed yield and its components in linseed. Different breeding strategy for crosswise as well as location wise is suggested because gene action was different in two locations.
\end{abstract}

Keywords: scaling tests, gene action, generation mean analysis, epistasis, linseed

\section{Introduction}

Linseed (Linum usitatissimum L.) is generally cultivated for either seed production or for fibre production. Linseed $(2 n=30)$ is one of the first crops domesticated by man. Every part of the linseed plant is utilized commercially either directly or after processing. Linseed is one of the important oilseed and fibre-yielding crops of India. India is the third largest producer in the world. However, the average national productivity $(403 \mathrm{Kg} / \mathrm{ha})$ is far below the world average $(851 \mathrm{Kg} / \mathrm{ha})$ due to non availability of high potential cultivars. The low productivity of flax in India is also attributed to its cultivation in marginal and sub-marginal, rainfed soils, lack of seed of improved varieties, meager or no application of fertilizers and it's sowing in utera cultivation. Nevertheless, due to narrow genetic base of the released varieties, concerted efforts are required to breed varieties involving distant and exotic germplasm, preferably from main centres of diversity, to produce varieties with higher level of productivity. Thus, creation of genetic variability involving diverse germplasm collections for yield and its contributing characters is of utmost importance for effective selection. In the present investigation, indigenous and exotic genotypes, diverse for yield and quality traits, have been used for the generation of breeding material. Qualitative characters controlled by one or a few major genes are more readily manipulated in a breeding programme as compared to quantitative traits controlled by many genes. Nevertheless, the breeder is concerned mainly with quantitative characteristics which could be of use in both formulating and performing the breeding programme. The inheritance of the characteristics chosen has a major influence on the strategy employed for cultivar development. Though the goals of linseed breeding programs vary considerably depending on the intended use, yet some traits considered important in most breeding program (Lay \& Dybing, 1989) such as yield where genetic improvement for yield and its components are most difficult to achieve due to the complex nature of their inheritance and influence of numerous environmental factors. The ability of the parents to combine well depends on the complex interactions among the genes adaptation which cannot be judged by mere yield performance and adaptation of the parents. Moreover, in a breeding programme an objective judgment about a particular cross combination likely to produce transgressive segregants in self 
pollinating crops like linseed would mainly depend upon the precise estimates of various components of genetic variances. An experimental method known as generation mean analysis (Anderson \& Kempthorne, 1954; Jinks \& Jones, 1958; Hayman, 1958) provides the opportunity to detect the presence or absence of epistasis and quantify them appropriately. In linseed, for formulating an efficient recombinant breeding programme, the required information on the nature of gene action, especially on the extent and kind of non-allelic interactions along with linkage among polygenes and gene dispersion in the parents of a cross with respect to yield and other economic traits is limited (Jinks, 1983). Hence, the present investigation has been carried out to study the nature of gene action for yield, fibre and quality traits in this crop.

\section{Materials and Methods}

The present investigation was carried out at the Experimental Farm of the Department of Crop Improvement, CSK HPKV Palampur HP (India) (E1) and Shivalik Extension and Research centre Kangra HP (E2); and offseason crossing work and generation advancement was done at High Altitude Extension and Research Centre, Kukumseri, HP India.

\subsection{Experimental Materials}

The experimental materials for gene action studies comprised of five diverse parents to develop three cross combinations. The parentage/source of these genotypes is given in Table 1 .

Table 1. Parentage/ source of genotypes used

\begin{tabular}{cll}
\hline S. No. & Genotypes & \multicolumn{1}{c}{ Parentage / source } \\
\hline 1 & Turkey & Exotic collection from Turkey \\
2 & Binwa & Flak-1 x SPS 47/7-10-3 \\
3 & TL-11 & Kiran x Solin, BARC ,Trombay \\
4 & Him Alsi- 2 & EC 21741 x LC216 \\
5 & TL-43 & Exotic collection from BARC, Trombay
\end{tabular}

\subsection{Layout of the Experiment}

The experimental material comprising of different generations viz., $\mathrm{P}_{1}, \mathrm{P}_{2}, \mathrm{~F}_{1}, \mathrm{~F}_{2}, \mathrm{BC}_{1}, \mathrm{BC}_{2}$ of three crosses were evaluated in Compact Family Block Design with three replications, during rabi 2008-2009 at two locations i.e Palampur (E1) and Kangra (E2). The row to row distance was $25 \mathrm{~cm}$ with plant to plant distance of $5 \mathrm{~cm}$ apart. The non segregating generations (Parents and $\mathrm{F}_{1}$ 's) were grown in single row of $2 \mathrm{~m}$ length. The segregating $\mathrm{F}_{2}$ generations were grown in six rows and $\mathrm{BC}_{1} \& \mathrm{BC}_{2}$ were grown in four rows.

\subsection{Recording of Observations}

The observations were recorded on 10 randomly taken plants per entry and per replication in case of parents and $\mathrm{F}_{1} \mathrm{~s}, 30$ in case of back crosses $\left(\mathrm{BC}_{1}, \mathrm{BC}_{2}\right)$ and 50 plants in case of $\mathrm{F}_{2}$ generation under both the sets of environment under study. Data on the basis of randomly taken competitive plants were recorded on 10 morphological characters, viz., days to $50 \%$ flowering, days to maturity, plant height $(\mathrm{cm})$, primary branches per plant, secondary branches per plant, capsules per plant, seeds per capsule, biological yield per plant (g), seed yield per plant $(\mathrm{g})$, harvest index $(\%)$ and 6 fibre characters viz., technical height $(\mathrm{cm})$, straw weight $(\mathrm{g})$, retted straw weight (g), line fibre (g), total fibre, percent fibre (\%) and 3 quality parameters viz., 1000-seed weight (g), oil content and protein content. Oil content of each genotype was determined by Nuclear Magnetic Resonance (NMR) at Oilseed Section, Department of Plant Breeding, PAU, Ludhiana and Crude Protein content (\%) was determined by Macro Kjeldahl method (AOAC, 1970) at Oilseed Section, Department of Plant Breeding, PAU, Ludhiana, India.

\subsection{Biometrical Analysis}

Estimation of Mather's simple scaling test (1949), joint scaling test of Cavalli (1952) and estimation of genic effects (Jinks \& Jones, 1958) were carried out as per the procedure given in detail by Mather and Jinks (1982) using SPAR1 programme (Doshi \& Gupta, 1991) at Department of Crop Improvement, CSK HPKV, Palampur, India. 


\subsection{Estimation of Genic Effects and Joint Scaling Test}

Estimation of various genic effects and test of fitness of appropriate genetic model was done according to joint scaling test of Cavalli (1952), as described in detail by Mather and Jinks (1982). Joint scaling test in general consists of estimating various genetic parameters from means of available type of generations followed by the comparison of observed generation means with the expected values, derived from the estimates of genetic parameters (genic effects), using weighted least square technique, taking as goodness of fit of a particular model was carried out by using weighted chi-square analysis. The observed and expected generation means were compared by $\chi^{2}$ test with the degree of parameters (p) estimated. In the present study, the estimation of genic effects and chi-square test of goodness of fit was carried out, using 3-parameters and 6 parameters models.

\section{Results and Discussion}

The nature and magnitude of genetic variation present in population is elucidated by genetic analysis of the quantitative traits. The predominance of additive gene effects signifies the development of homozygous lines while dominance gene effects are used for exploitation of hybrid vigour. The generation mean is the most common tool employed for the estimation of gene effect and components of genetic variance (Hayman \& Mather, 1955).The additive and dominance gene effects along with epistatic interactions were estimated for yield and its component traits in three crosses. In case of non-significance of scaling tests, the data of different traits, were subjected to joint scaling test of Cavalli (1952). Non significant chi-square value indicated adequacy of three parameter model, but in case of significant chi-square value, the analysis was further extended to the epistatic model (six parameter model). The results of scaling tests (Table $4 \& 5$ ) revealed that additive dominance model was fit in cross Turkey x Binwa for biological yield per plant, straw weight, retted straw weight, total fibre, line fibre, seeds per capsule, seed yield per plant in E1; seed yield per plant in cross TL-43 x Binwa (E2), which suggested the absence of epistasis in these crosses. Dominance component was found to be predominant over additive component hence breeding strategy in above crosses should be hybridization followed by deffering selection to later generations. The above model was a failure thereby indicating presence of non allelic interactions for remaining traits in all the crosses.

Gene interaction is considered to be complementary when the [d] and [dd] estimates have the same signs and to be duplicating when the signs differ (Mather \& Jinks, 1982).Complementary type of gene action was observed for biological yield per plant and seed yield per plant in E1, biological yield per plant in E2 in cross TL-11 x Him Alsi-2; seed yield per plant and plant height in E1 (Table 2 \& 3), days to maturity and plant height in E2 in cross Turkey $\mathrm{x}$ Binwa, days to 50 per cent flowering and biological yield per plant in cross TL-43 x Binwa in E2 (Table 7). Such type of gene action was not observed for remaining traits in all the crosses. The traits for which the above gene action was observed, the breeding strategy should be selection in early generation by adopting biparental matings for isolation of transgressive segregants. Patel et al. (1997) reported the preponderance of additive components for phenological traits. Duplicate type of gene action was observed for days to 50 per cent flowering, plant height, technical height in E1 and days to 50 per cent flowering, seed yield per plant, plant height, technical height, straw weight, retted straw weight, total fibre, per cent fibre, line fibre in E2 in cross TL-11 x Him Alsi-2; technical height in both E1 and E2 in cross Turkey x Binwa, secondary branches per plant, 1000 seed weight, plant height in E1 (Table 6) and technical height in E2 in cross TL-43 x Binwa. Duplicate type of gene action was not observed for the remaining traits in all the crosses. The traits for which the above gene action was observed, the breeding strategy would be to grow large segregating populations and adopting biparental mating to get transgressive segregants. The prevalence of duplicate epistasis in the inheritance of certain traits including seed yield indicated that these traits might be improved through recurrent selection in biparental progenies that would help in exploiting the duplicate type of non-allelic interaction and allow recombination and concentration of genes having cumulative effects in population as this method is helpful in breaking up undesirable linkages as reported by Ganesh and Sakila (1999). Gauraha and Rao (2011) reported that duplicate type of epistasis played a major role in the expression of most of the characters studied for various crosses in linseed which is in accordance with our findings. Tyson (1989) reported that dominance gene action and maternal effects contributed in genetic variation of seed weight in flax. Singh et al (2006) and Sood et al. (2007) have reported additive gene action. Tak (1996) revealed that seed yield was mainly controlled by duplicate type of gene effects. 
Table 2. Estimates of scaling tests and genic effects in linseed for cross TL-11 x Him Alsi-2

\begin{tabular}{|c|c|c|c|c|c|c|c|c|c|c|c|}
\hline Parameters & Locations & $\begin{array}{c}50 \% \\
\text { flowering }\end{array}$ & $\begin{array}{l}\text { Days to } \\
\text { maturity }\end{array}$ & $\begin{array}{c}\text { Primary } \\
\text { branches per } \\
\text { plant }\end{array}$ & $\begin{array}{c}\text { Secondary } \\
\text { branches per } \\
\text { plant }\end{array}$ & $\begin{array}{c}\text { Capsules per } \\
\text { plant }\end{array}$ & $\begin{array}{l}\text { Seeds per } \\
\text { capsule }\end{array}$ & $\begin{array}{c}\text { Biological } \\
\text { yield per plant }\end{array}$ & $\begin{array}{l}\text { Seed yield per } \\
\text { plant }\end{array}$ & Harvest Index & $\begin{array}{l}\text { 1000-seed } \\
\text { weight }\end{array}$ \\
\hline \multirow[t]{2}{*}{ A } & E1 & $-1.26^{* *} \pm 0.44$ & $8.50 * * \pm 0.50$ & $-1.16^{* *} \pm 0.30$ & $-1.78^{* *} \pm 0.25$ & $7.55^{* *} \pm 1.08$ & $1.12^{* *} \pm 0.38$ & $-3.03 * * \pm 0.28$ & $-1.59 * * \pm 0.14$ & $0.39 \pm 2.56$ & $0.89^{* *} \pm 0.06$ \\
\hline & E2 & $1.42 * * \pm 0.47$ & $7.74 * * \pm 0.64$ & $-1.26^{* *} \pm 0.29$ & $-1.78^{* *} \pm 0.21$ & $7.45^{* *} \pm 1.06$ & $1.62 * * \pm 0.36$ & $-3.18^{* * \pm 0.24}$ & $-1.19^{* *} \pm 0.14$ & $1.00 \pm 2.86$ & $1.26^{* *} \pm 0.08$ \\
\hline \multirow[t]{2}{*}{ B } & E1 & $-0.40 \pm 0.39$ & $5.74 * * \pm 0.60$ & $-2.20^{* *} \pm 0.41$ & $-1.36^{* *} \pm 0.26$ & $-8.03^{* *} \pm 1.26$ & $1.56^{* * \pm 0.37}$ & $1.61 * * \pm 0.24$ & $0.60^{* *} \pm 0.14$ & $-1.87 \pm 1.80$ & $0.57 \pm 0.05$ \\
\hline & E2 & $1.00 * \pm 0.44$ & $5.18^{* *} \pm 0.71$ & $-2.06^{* *} \pm 0.40$ & $-1.73^{* *} \pm 0.21$ & $-8.16 \pm 1.27$ & $1.22 * * \pm 0.33$ & $0.43 \pm 0.25$ & $1.05^{* *} \pm 0.18$ & $11.07^{* *} \pm 2.81$ & $0.56^{* *} \pm 0.06$ \\
\hline \multirow[t]{2}{*}{$\mathrm{C}$} & E1 & $-8.83^{* *} \pm 0.67$ & $9.56^{* *} \pm 1.03$ & $-1.03 \pm 0.62$ & $-3.60^{* *} \pm 0.48$ & $-3.23 \pm 2.59$ & $4.20^{* * \pm 0.69}$ & $4.74 * * \pm 0.33$ & $4.62^{* *} \pm 0.30$ & $32.52 * * \pm 2.34$ & $0.51^{* *} \pm 0.09$ \\
\hline & E2 & $6.56^{* *} \pm 0.75$ & $9.86^{* *} \pm 1.23$ & $-1.00 \pm 0.60$ & $-3.96^{* *} \pm 0.38$ & $-3.46 \pm 2.58$ & $-0.26 \pm 0.60$ & $2.52^{* *} \pm 0.46$ & $3.27^{* *} \pm 0.25$ & $30.84 * * \pm 4.06$ & $0.89^{* *} \pm 0.13$ \\
\hline \multirow[t]{2}{*}{ D } & E1 & $-3.58^{* *} \pm 0.21$ & $-2.33^{* *} \pm 0.22$ & $1.16^{* *} \pm 0.26$ & $-0.22 \pm 0.17$ & $-1.37 \pm 1.36$ & $0.75^{*} \pm 0.29$ & $3.08^{* *} \pm 0.17$ & $2.80^{* *} \pm 0.13$ & $17.00^{* *} \pm 1.44$ & $0.09^{*} \pm 0.03$ \\
\hline & E2 & $3.07^{* *} \pm 0.28$ & $1.53 * * \pm 0.28$ & $1.16^{* *} \pm 0.26$ & $-0.22 \pm 0.17$ & $-1.37 \pm 1.36$ & $-1.55^{* *} \pm 0.25$ & $2.63^{* *} \pm 0.16$ & $1.70^{* *} \pm 0.10$ & $9.38^{* *} \pm 1.51$ & $0.09 \pm 0.05$ \\
\hline \multirow[t]{2}{*}[\mathrm{m}]{} & E1 & $107.75^{* *} \pm 0.43$ & $162.52^{* *} \pm 0.48$ & $6.80^{* *} \pm 0.53$ & $2.66^{* *} \pm 0.07$ & $20.66^{* *} \pm 0.32$ & $8.35^{* *} \pm 0.14$ & $11.35^{* *} \pm 0.35$ & $7.77 * * \pm 0.27$ & $73.48 * * 2.67$ & $7.63^{* *} \pm 0.03$ \\
\hline & E2 & $98.74 \pm 0.58$ & $149.13^{* *} \pm 0.59$ & $6.90 * * \pm 0.53$ & $2.58^{* *} \pm 0.08$ & $21.15^{* *} \pm 0.32$ & $3.72^{* *} \pm 0.50$ & $10.79^{* *} \pm 0.34$ & $5.31^{* *} \pm 0.21$ & $52.45^{* *} \pm 1.59$ & $7.27^{* *} \pm 0.02$ \\
\hline \multirow[t]{2}{*}{ [d] } & E1 & $2.78^{* *} \pm 0.09$ & - & - & - & $2.88^{* *} \pm 0.36$ & - & - & - & $4.42^{* *} \pm 0.61$ & $0.89^{* *} \pm 0.02$ \\
\hline & E2 & $2.66 \pm 0.09$ & - & - & $0.20^{* *} \pm 0.06$ & $2.89^{* *} \pm 0.36$ & - & - & - & $3.14^{* *} \pm 1.17$ & $0.96^{* *} \pm 0.02$ \\
\hline \multirow[t]{2}{*}{ [h] } & E1 & $11.03^{* *} \pm 1.21$ & $21.90^{* *} \pm 1.26$ & $-7.31^{* *} \pm 1.36$ & $-3.00^{* *} \pm 0.15$ & $5.75^{* *} \pm 0.54$ & - & $10.74 * * \pm 0.97$ & $10.92 * * \pm 0.64$ & $63.77 * * \pm 7.58$ & $0.16^{* *} \pm 0.05$ \\
\hline & E2 & $9.86 \pm 1.57$ & $16.90^{* *} \pm 1.61$ & $-7.50^{* *} \pm 1.35$ & $-3.03^{* *} \pm 0.32$ & $4.83^{* *} \pm 0.53$ & $9.18^{* *} \pm 1.27$ & $10.19^{* *} \pm 0.85$ & $-5.26^{* *} \pm 0.57$ & $13.20^{* *} \pm 2.78$ & $1.13^{* *} \pm 0.11$ \\
\hline \multirow[t]{2}{*}{ [i] } & E1 & $7.16 \pm 0.42$ & $4.67 * * \pm 0.45$ & $-2.33^{* *} \pm 0.52$ & $0.44 \pm 0.35$ & $2.75 \pm 2.72$ & $-1.51^{*} \pm 0.58$ & $-6.17 * * \pm 0.34$ & $-5.61 * * \pm 0.27$ & $-34.00^{* *} \pm 2.88$ & $-0.18^{* *} \pm 0.07$ \\
\hline & E2 & $6.14^{* *} \pm 0.57$ & $3.06^{* *} \pm 0.57$ & $-2.33^{* *} \pm 0.52$ & $0.44 \pm 0.35$ & $2.75 \pm 2.72$ & $3.11^{* *} \pm 0.50$ & $-5.27 * * \pm 0.33$ & $-3.40^{* *} \pm 0.21$ & $-18.76^{* *} \pm 3.02$ & $-0.19 \pm 0.11$ \\
\hline \multirow[t]{2}{*}{ [j] } & E1 & $-0.43 * \pm 0.20$ & $1.37 * * \pm 0.23$ & $0.51^{* *} \pm 0.21$ & $-0.21 \pm 0.13$ & $7.79 * * \pm 0.78$ & $-0.22 \pm 0.22$ & $-2.32 * * \pm 0.17$ & $-1.09 * * \pm 0.09$ & $1.13 \pm 1.48$ & $0.73^{* *} \pm 0.03$ \\
\hline & E2 & $-1.21^{* *} \pm 0.24$ & $1.27 * * \pm 0.28$ & $0.40 \pm 0.20$ & $-0.02 \pm 0.13$ & $7.81^{* *} \pm 0.78$ & $0.20 \pm 0.19$ & $-1.80 * * \pm 0.12$ & $-1.12 * * \pm 0.10$ & $-5.03 \pm 1.73$ & $0.91 * * \pm 0.04$ \\
\hline \multirow[t]{2}{*}{ [1] } & E1 & $-5.50^{* *} \pm 0.98$ & $-18.92^{* *} \pm 1.16$ & $5.70 * * \pm 0.93$ & $2.71^{* *} \pm 0.62$ & $-2.27 \pm 3.79$ & $-1.17 \pm 0.98$ & $7.60^{* *} \pm 0.66$ & $6.60^{* *} \pm 0.39$ & $35.48 * \pm 5.74$ & $-0.13 \pm 0.13$ \\
\hline & E2 & $-5.72 * * \pm 1.15$ & $-16.00^{* *} \pm 1.47$ & $5.66^{* *} \pm 0.92$ & $3.07 * * \pm 0.55$ & $-2.04 \pm 3.78$ & $-5.95 * * \pm 0.89$ & $8.01 * * \pm 0.62$ & $3.54 * * \pm 0.39$ & $6.69 \pm 6.50$ & $-0.49 * \pm 0.19$ \\
\hline \multirow[t]{2}{*}{$\chi^{2}$} & E1 & $4.33^{*}$ & 3.44 & 0.49 & 1.60 & 1.56 & 1.29 & $68.12^{* *}$ & $78.85^{* *}$ & 0.58 & 0.98 \\
\hline & E2 & $24.63^{* *}$ & 3.44 & 0.10 & 1.71 & 1.80 & 3.39 & $134.71^{* *}$ & $51.85^{* *}$ & 1.05 & 3.16 \\
\hline \multirow{2}{*}{$\begin{array}{l}\text { Type of } \\
\text { interaction }\end{array}$} & E1 & $\mathrm{D}$ & - & - & - & - & - & $\mathrm{C}$ & $\mathrm{C}$ & - & - \\
\hline & E2 & $\mathrm{D}$ & - & - & - & - & - & $\mathrm{C}$ & $\mathrm{D}$ & - & - \\
\hline
\end{tabular}

Table 3. Estimates of scaling tests and genic effects in linseed for cross TL-11 x Him Alsi-2

\begin{tabular}{|c|c|c|c|c|c|c|c|c|c|c|}
\hline Parameters & Locations & Plant height & $\begin{array}{c}\text { Technical } \\
\text { height }\end{array}$ & Straw weight & $\begin{array}{c}\text { Retted straw } \\
\text { weight }\end{array}$ & Total fibre & Percent fibre & Line fibre & Oil content & $\begin{array}{l}\text { Protein } \\
\text { content }\end{array}$ \\
\hline & E1 & $12.78 * * \pm 0.90$ & $-2.19 \pm 1.36$ & $-0.56^{* *} \pm 0.12$ & $-0.64 * * \pm 0.12$ & $-0.12 * * \pm 0.02$ & $-0.53^{* *} \pm 0.16$ & $-0.08^{* *} \pm 0.01$ & $-3.50 * * \pm 0.69$ & $2.45^{* *} \pm 0.41$ \\
\hline & E2 & $15.05^{* *}{ }_{ \pm 0.86}$ & $-1.16 \pm 0.69$ & $-1.18 * * \pm 0.16$ & $-1.18^{* *} \pm 0.16$ & $-0.23^{* *} \pm 0.03$ & $-2.07 * * \pm 0.45$ & $-0.15^{* *} \pm 0.02$ & - & - \\
\hline \multirow[t]{2}{*}{ B } & E1 & $14.41 * * \pm 1.17$ & $16.27 * * \pm 1.38$ & $1.19 * * \pm 0.19$ & $1.11 * *_{ \pm} 0.20$ & $0.22 * * \pm 0.04$ & $0.64 * * \pm 0.15$ & $0.15 * * \pm 0.02$ & $-4.40 * * \pm 0.75$ & $1.39 * * \pm 0.37$ \\
\hline & E2 & $5.11 * * \pm 1.35$ & $17.27 * * \pm 0.98$ & $0.20 \pm 0.13$ & $0.20 \pm 0.13$ & $0.04 \pm 0.02$ & $0.11 \pm 0.14$ & $0.02 \pm 0.01$ & - & - \\
\hline $\mathrm{C}$ & E1 & $28.75^{* *} \pm 1.63$ & $-3.71 \pm 2.55$ & $0.44 * \pm 0.21$ & $0.09 \pm 0.20$ & $0.01 \pm 0.04$ & $0.29 \pm 0.22$ & $0.01 \pm 0.02$ & $-3.50 * * \pm 1.21$ & $3.22 * * \pm 0.66$ \\
\hline \multirow[t]{2}{*}{ D } & E1 & $15.19 * * \pm 0.30$ & $-8.90 * * \pm 0.32$ & $-0.09 \pm 0.09$ & $-0.18 \pm 0.10$ & $-0.03 \pm 0.02$ & $0.09 \pm 0.09$ & $-0.02 \pm 0.01$ & $2.20^{* *} \pm 0.48$ & $3.53 * * \pm 0.27$ \\
\hline & E2 & $15.19 * * \pm 0.30$ & $-8.90 * * \pm 0.32$ & $0.96 * * \pm 0.13$ & $0.96 * * \pm 0.13$ & $0.19^{* *} \pm 0.02$ & $1.30 * * \pm 0.24$ & $0.12 * * \pm 0.01$ & - & - \\
\hline \multirow[t]{2}{*}[\mathrm{m}]{} & E1 & $35.49 * * \pm 0.89$ & $21.06 * * \pm 0.81$ & $3.00 * * \pm 0.07$ & $2.83 * *_{ \pm 0} .05$ & $0.49 * * \pm 0.01$ & $17.44^{* *} \pm 0.05$ & $0.33 * *_{ \pm} 0.00$ & $44.26 * 6_{ \pm} .01$ & $13.77 \pm 0.57$ \\
\hline & E2 & $37.98 \pm 0.84$ & $21.70 * * \pm 0.75$ & $5.15^{* *} \pm 0.28$ & $4.63 * * \pm 0.28$ & $0.85^{* *} \pm 0.05$ & $19.94 * * \pm 0.48$ & $0.56 * * \pm 0.03$ & - & - \\
\hline \multirow[t]{2}{*}{ [d] } & E1 & - & - & $0.19 * * \pm 0.07$ & $0.18 * \pm 0.07$ & $0.03 * \pm 0.01$ & $0.13 * \pm 0.07$ & $0.02 * \pm 0.00$ & - & - \\
\hline & E2 & - & - & - & - & - & - & - & - & - \\
\hline$[\mathrm{h}]$ & E1 & $59.52 * * \pm 2.43$ & $53.71 * * \pm 2.43$ & $1.04 * * \pm 0.24$ & $0.50 * *_{ \pm} 0.08$ & $0.10^{* *} \pm 0.01$ & $0.46^{* *} \pm 0.09$ & $0.06 * * \pm 0.01$ & $-18.60 * * \pm 2.56$ & $17.28 \pm 1.45$ \\
\hline \multirow[t]{2}{*}{ [i] } & E1 & $30.38 * * \pm 0.61$ & $17.80 * * \pm 0.64$ & $0.18 \pm 0.18$ & $0.37 \pm 0.20$ & $0.07 \pm 0.04$ & $-0.18 \pm 0.19$ & $0.05 \pm 0.02$ & $-4.40 * * \pm 0.96$ & $7.06^{* *} \pm 0.96$ \\
\hline & E2 & $30.38 * * \pm 0.61$ & $17.80 * * \pm 0.64$ & $-1.92 * * \pm 0.27$ & $-1.92 * * \pm 0.27$ & $-0.38 * * \pm 0.05$ & $-2.60^{* *} \pm 0.48$ & $-0.24 * * \pm 0.03$ & - & - \\
\hline \multirow[t]{2}{*}{ [j] } & E1 & $-13.59 * * \pm 0.69$ & $-9.23 * * \pm 0.53$ & $-0.88^{* *} \pm 0.10$ & $-0.88^{* *} \pm 0.11$ & $-0.17 * * \pm 0.02$ & $-0.59 * * \pm 0.10$ & $-0.11 * * \pm 0.01$ & $0.45 \pm 0.44$ & $0.53^{*} \pm 0.24$ \\
\hline & E2 & $-13.99 * * \pm 0.71$ & $-9.22 * * \pm 0.50$ & $0.69 * * \pm 0.10$ & $-0.69 * * \pm 0.10$ & $-0.13 * * \pm 0.02$ & $-1.09 * * \pm 0.23$ & $-0.08 * * \pm 0.01$ & - & - \\
\hline \multirow[t]{2}{*}[1]{} & E1 & $-32.01 * *_{ \pm} 1.75$ & $-31.88^{* *} \pm 2.66$ & $-0.81 * \pm 0.37$ & $-0.85 * \pm 0.40$ & $-0.17 * \pm 0.08$ & $0.08 \pm 0.37$ & $-0.11 * \pm 0.05$ & $12.30 * *_{ \pm} .72$ & $-10.90^{* *} \pm 0.96$ \\
\hline & E2 & $-28.26^{* *} \pm 1.93$ & $-33.91 * *_{ \pm} 1.61$ & $2.90 * * \pm 0.43$ & $2.90 * * \pm 0.43$ & $0.57^{* *} \pm 0.08$ & $4.56^{* *} \pm 0.92$ & $0.37 * * \pm 0.05$ & - & - \\
\hline \multirow[t]{2}{*}{$\chi^{2}$} & E1 & $44.61 * *$ & $10.41 * *$ & 0.99 & 4.45 & 4.47 & 2.05 & 4.48 & 1.99 & 0.68 \\
\hline & E2 & $4.30^{*}$ & $11.90^{* *}$ & $37.49^{* *}$ & $37.40^{* *}$ & $36.75^{* *}$ & $19.46^{* *}$ & $36.15^{* *}$ & - & - \\
\hline Type of & E1 & $\mathrm{D}$ & $\mathrm{D}$ & - & - & - & - & - & - & - \\
\hline interaction & E2 & $\mathrm{D}$ & $\mathrm{D}$ & $\mathrm{D}$ & $\mathrm{D}$ & $\mathrm{D}$ & $\mathrm{D}$ & $\mathrm{D}$ & - & - \\
\hline${ }^{*} \mathrm{P}=0.05 ;$ and & & & & & & & & & & \\
\hline
\end{tabular}


Table 4. Estimates of scaling tests and genic effects in linseed for cross Turkey x Binwa

\begin{tabular}{|c|c|c|c|c|c|c|c|c|c|c|c|}
\hline $\begin{array}{l}\text { Traits } \\
\text { Parameters }\end{array}$ & Locations & $\begin{array}{l}50 \text { per cent } \\
\text { flowering }\end{array}$ & $\begin{array}{l}\text { Days to } \\
\text { maturity }\end{array}$ & $\begin{array}{l}\text { Primary } \\
\text { branches } \\
\text { per plant }\end{array}$ & $\begin{array}{c}\text { Secondary } \\
\text { branches per } \\
\text { plant }\end{array}$ & $\begin{array}{l}\text { Capsules per } \\
\text { plant }\end{array}$ & $\begin{array}{l}\text { Seeds per } \\
\text { capsule }\end{array}$ & $\begin{array}{c}\text { Biological } \\
\text { yield per } \\
\text { plant }\end{array}$ & $\begin{array}{l}\text { Seed yield } \\
\text { per plant }\end{array}$ & Harvest Index & $\begin{array}{l}\text { 1000-seed } \\
\text { weight }\end{array}$ \\
\hline \multirow[t]{2}{*}{ A } & E1 & $1.41 * * \pm 0.43$ & $0.20 \pm 0.32$ & $3.67 * * \pm 0.32$ & $0.83 * * \pm 0.25$ & $3.93 * \pm 1.65$ & $0.31 \pm 0.43$ & $0.12 \pm 0.24$ & $0.11 \pm 0.11$ & $0.28 \pm 2.08$ & $-0.20 \pm 0.11$ \\
\hline & E2 & $1.81^{* *} \pm 0.45$ & $-0.88^{*} \pm 0.34$ & $3.64^{* *} \pm 0.30$ & $0.80^{* *} \pm 0.23$ & $5.80^{* *} \pm 1.53$ & $0.21 \pm 0.41$ & $0.25 \pm 0.22$ & $0.14 \pm 0.10$ & $1.67 \pm 2.02$ & $0.21 * \pm 0.08$ \\
\hline \multirow[t]{2}{*}{ B } & E1 & $2.38^{* *} \pm 0.31$ & $30.64 * * \pm 0.33$ & $-3.14^{* *} \pm 0.39$ & $-0.81 * * \pm 0.21$ & $15.22 * * \pm 1.22$ & $-0.82 * \pm 0.38$ & $0.40 \pm 0.25$ & $0.29^{* *} \pm 0.10$ & $2.64 \pm 1.79$ & $0.27 * * \pm 0.06$ \\
\hline & E2 & $2.32 * * \pm 0.38$ & $-30.71^{* *} \pm 0.36$ & $-3.04 * * \pm 0.37$ & $-0.97^{* *} \pm 0.20$ & $14.25^{* *} \pm 1.22$ & $-0.02 \pm 0.39$ & $0.18 \pm 0.21$ & $0.89^{* *} \pm 0.06$ & $15.65^{* *} \pm 1.41$ & $0.32 * * \pm 0.05$ \\
\hline \multirow[t]{2}{*}{$\mathrm{C}$} & E1 & $6.70^{* *} \pm 0.49$ & $30.36^{* *} \pm 0.56$ & $-5.86^{* *} \pm 0.58$ & $-2.13^{* *} \pm 0.39$ & $22.73^{* *} \pm 1.77$ & $-4.90^{* *} \pm 0.65$ & $0.56 \pm 0.42$ & $2.27 * * \pm 0.30$ & $10.46^{* *} \pm 3.23$ & $0.51^{* *} \pm 0.15$ \\
\hline & E2 & $6.46^{* *} \pm 0.66$ & $-30.33^{* *} \pm 0.59$ & $-5.73^{* *} \pm 0.56$ & $-2.26^{* *} \pm 0.38$ & $19.90^{* *} \pm 1.67$ & $-1.56^{* *} \pm 0.49$ & $1.13^{* *} \pm 0.38$ & $1.48^{* *} \pm 0.11$ & $21.81^{*} \pm 2.71$ & $0.55^{* *} \pm 0.13$ \\
\hline \multirow[t]{2}{*}{$\mathrm{D}$} & E1 & $1.45^{* *} \pm 0.24$ & $0.03 \pm 0.21$ & $0.47 \pm 0.24$ & $0.24 \pm 0.17$ & $5.72^{* *} \pm 1.17$ & $1.88^{* *} \pm 0.35$ & $0.14 \pm 0.17$ & $1.04 * * \pm 0.13$ & $4.04 * * \pm 1.21$ & $0.22 * * \pm 0.07$ \\
\hline & E2 & $1.16^{* *} \pm 0.30$ & $0.63^{* *} \pm 0.23$ & $0.47 \pm 0.24$ & $0.24 \pm 0.17$ & $5.72 * * \pm 1.17$ & $0.66^{*} \pm 0.28$ & $0.34 * \pm 0.17$ & $0.21 * *_{ \pm 0} .04$ & $2.24 \pm 1.18$ & $0.22 * * \pm 0.07$ \\
\hline \multirow[t]{2}{*}[\mathrm{m}]{} & E1 & $115.19^{* *} \pm 0.05$ & $183.06 \pm 0.14$ & $6.22^{* *} \pm 0.51$ & $2.99^{* *} \pm 0.09$ & $15.34^{* *} *_{ \pm 0.72}$ & $3.88^{* *} \pm 0.72$ & $5.45^{* *} \pm 0.08$ & $3.78^{* *} \pm 0.27$ & $35.58 * * \pm 0.32$ & $7.17^{* *} \pm 0.05$ \\
\hline & E2 & $104.97^{* *} \pm 0.08$ & $167.96^{* *} \pm 0.15$ & $6.16^{* * \pm 0.51}$ & $3.07 * * \pm 0.09$ & $15.51 * * \pm 0.72$ & $6.71 * * \pm 0.06$ & $5.52 \pm 0.04$ & $2.07 * * \pm 0.01$ & $39.02 * * \pm 0.54$ & $7.16^{* *} \pm 0.05$ \\
\hline \multirow[t]{2}{*}{ [d] } & E1 & $-0.83^{* *} \pm 0.14$ & $15.43 \pm 0.14$ & $-0.41^{* *} \pm 0.11$ & $0.32 * * \pm 0.07$ & $-7.06^{* *} \pm 0.44$ & $-0.80 * * \pm 0.11$ & $-0.39 * * \pm 0.07$ & - & - & $-0.68^{* *} \pm 0.04$ \\
\hline & E2 & $-0.95^{* *} \pm 0.11$ & $15.33^{* *} \pm 0.15$ & $-0.39 * * \pm 0.11$ & $0.26 * * \pm 0.06$ & $-7.48^{* *} \pm 0.34$ & $-0.52 * * \pm 0.11$ & $-0.24 \pm 0.06$ & $0.08 * \pm 0.03$ & $3.247^{* *} \pm 0.80$ & $0.63 * * \pm 0.02$ \\
\hline \multirow[t]{2}{*}[\mathrm{h}]{} & E1 & - & $45.58 \pm 0.52$ & $-9.03^{* *} \pm 1.30$ & $-2.58 * * \pm 0.35$ & $12.89^{* *} \pm 0.81$ & $5.54^{* *} \pm 1.82$ & $0.50^{* *} \pm 0.15$ & $-3.63^{* *} \pm 0.59$ & - & $-0.75^{* *} \pm 0.07$ \\
\hline & E2 & - & $46.33^{* *} \pm 0.54$ & $-8.87^{* *} \pm 1.28$ & $-2.82 * * \pm 0.33$ & $12.73^{* *} \pm 0.81$ & - & - & - & - & $0.75^{* *} \pm 0.07$ \\
\hline \multirow[t]{2}{*}{ [i] } & E1 & $-2.90 * \pm 0.49$ & $-0.07 \pm 0.43$ & $-0.95 \pm 0.48$ & $0.48 \pm 0.34$ & $11.44^{* *} \pm 2.34$ & $3.76^{* *} \pm 0.71$ & $-0.28 \pm 0.35$ & $-2.09^{* *} \pm 0.27$ & $-8.08^{* *} \pm 2.42$ & $-0.44^{* *} \pm 0.14$ \\
\hline & E2 & $-2.33 * \pm 0.61$ & $-1.26 * * 0.46$ & $-0.95 \pm 0.48$ & $0.48 \pm 0.34$ & $11.44 * * \pm 2.34$ & $1.33 * \pm 0.57$ & $-0.69 * \pm 0.34$ & $-0.43^{* *} \pm 0.09$ & $-4.49 \pm 2.37$ & $-0.44 * * \pm 0.14$ \\
\hline \multirow[t]{2}{*}{ [j] } & E1 & $-0.48 * \pm 0.24$ & $15.42 * * \pm 0.20$ & $-0.26 \pm 0.23$ & $-0.01 \pm 0.14$ & $9.57^{* *} \pm 1.01$ & $0.25 \pm 0.27$ & $-0.26 \pm 0.15$ & $-0.20 \pm 0.05$ & $-1.47 \pm 1.07$ & $-0.23^{* *} \pm 0.06$ \\
\hline & E2 & $-0.25 \pm 0.26$ & $14.91 * * \pm 0.21$ & $-0.30 \pm 0.22$ & $0.08 \pm 0.14$ & $10.02 * * \pm 0.97$ & $-0.09 \pm 0.26$ & $0.03 \pm 0.13$ & $-0.37^{* *} \pm 0.05$ & $-6.98^{* *} \pm 1.14$ & $-0.26^{* *} \pm 0.04$ \\
\hline \multirow[t]{2}{*}{ [1] } & E1 & $-0.90 \pm 0.93$ & $30.52 * * \pm 0.79$ & $7.77 * * \pm 0.85$ & $1.15 * \pm 0.58$ & $-0.15 \pm 4.06$ & $-2.63 * \pm 1.14$ & $-0.00 \pm 0.61$ & $1.91^{* *} \pm 0.35$ & $5.70 \pm 4.72$ & $0.38 \pm 0.22$ \\
\hline & E2 & $-1.80 \pm 1.09$ & $32.86 * * \pm 0.84$ & $7.64 * * \pm 0.83$ & $1.28 * \pm 0.57$ & $-2.98 \pm 4.01$ & $-1.10 \pm 1.06$ & $0.26 \pm 0.56$ & $-0.60 * * \pm 0.20$ & $-12.82 * * \pm 4.22$ & $0.34 \pm 0.20$ \\
\hline \multirow[t]{2}{*}{$\chi^{2}$} & E1 & 0.95 & 0.03 & 1.27 & 2.05 & 0.00 & 0.87 & 4.08 & $5.71^{*}$ & $9.84^{*}$ & 2.87 \\
\hline & E2 & 4.88 & $7.42^{* *}$ & 1.79 & 2.29 & 0.55 & 3.34 & 0.77 & 3.52 & 2.57 & 2.68 \\
\hline \multirow{2}{*}{$\begin{array}{l}\text { Type of } \\
\text { interaction }\end{array}$} & E1 & - & - & - & - & - & - & - & $\mathrm{C}$ & - & - \\
\hline & E2 & - & $\mathrm{C}$ & - & - & - & - & - & - & - & - \\
\hline${ }^{*} \mathrm{P}=\mathbf{0 . 0 5}$; an & $P=0.01$ & & & & & & & & & & \\
\hline
\end{tabular}

Table 5. Estimates of scaling tests and genic effects in linseed for cross Turkeyx Binwa

\begin{tabular}{|c|c|c|c|c|c|c|c|c|c|c|}
\hline Parameters & Locations & Plant height & $\begin{array}{l}\text { Technical } \\
\text { height }\end{array}$ & Straw weight & $\begin{array}{l}\text { Retted straw } \\
\text { weight }\end{array}$ & Total fibre & Percent fibre & Line fibre & Oil content & Protein content \\
\hline \multirow[t]{2}{*}{ A } & E1 & $2.13^{* *} \pm 0.55$ & $0.81 \pm 1.30$ & $0.05 \pm 0.23$ & $0.05 \pm 0.23$ & $0.00 \pm 0.04$ & $0.19 \pm 0.25$ & $0.00 \pm 0.03$ & $0.30 \pm 0.69$ & $2.25 * * \pm 0.40$ \\
\hline & E2 & $-1.53^{* *} \pm 0.55$ & $2.31 \pm 1.33$ & $0.56^{* *} \pm 0.21$ & $0.87^{* *} \pm 0.21$ & $0.17^{* *} \pm 0.04$ & $1.06^{* * \pm 0.32}$ & $0.12^{* *} \pm 0.02$ & - & - \\
\hline \multirow[t]{2}{*}{$\mathrm{B}$} & E1 & $6.70 * * \pm 1.27$ & $5.15 * * \pm 0.83$ & $0.19 \pm 0.23$ & $0.36 \pm 0.22$ & $0.07 \pm 0.04$ & $0.35^{*} \pm 0.17$ & $0.05 \pm 0.02$ & $1.10 \pm 0.70$ & $0.19 \pm 0.37$ \\
\hline & E2 & $8.53^{* *} \pm 1.07$ & $6.05^{* *} \pm 0.83$ & $-0.43^{*} \pm 0.19$ & $-0.12 \pm 0.19$ & $-0.02 \pm 0.03$ & $0.14 \pm 0.17$ & $-0.01 \pm 0.02$ & - & - \\
\hline \multirow[t]{2}{*}{$\mathrm{C}$} & E1 & $17.26^{* *} \pm 1.48$ & $10.66^{* *} \pm 2.78$ & $-0.00 \pm 0.40$ & $0.16 \pm 0.39$ & $0.03 \pm 0.07$ & $0.12 \pm 0.30$ & $0.02 \pm 0.05$ & $2.20 \pm 1.17$ & $3.46^{* *} \pm 0.69$ \\
\hline & E2 & $19.70^{* *} \pm 1.31$ & $-8.26^{* *} \pm 1.76$ & $0.40 \pm 0.36$ & $1.02^{* *} \pm 0.36$ & $0.20^{* *} \pm 0.07$ & $1.45^{* *} \pm 0.35$ & $0.13^{* *} \pm 0.04$ & - & - \\
\hline \multirow[t]{2}{*}{$\mathrm{D}$} & E1 & $6.35^{* *} \pm 0.47$ & $8.31^{* *} \pm 1.54$ & $0.12 \pm 0.16$ & $0.12 \pm 0.16$ & $0.02 \pm 0.03$ & $0.02 \pm 0.14$ & $0.01 \pm 0.02$ & $1.50^{* * \pm 0.51}$ & $0.51 \pm 0.28$ \\
\hline & $\mathrm{E} 2$ & $6.35 * * \pm 0.47$ & $8.31 * * \pm 1.08$ & $0.14 \pm 0.16$ & $0.14 \pm 0.16$ & $0.02 \pm 0.03$ & $0.12 \pm 0.14$ & $0.01 \pm 0.02$ & - & - \\
\hline \multirow[t]{2}{*}[\mathrm{m}]{} & E1 & $77.90 * * \pm 0.51$ & $28.28^{* *} \pm 3.00$ & $3.27 * * \pm 0.07$ & $3.00 \pm 0.07$ & $0.53^{* *} \pm 0.01$ & $17.56^{* *} \pm 0.06$ & $0.35^{* *} \pm 0.01$ & $42.03 * * \pm 1.05$ & $20.89^{* *} \pm 0.14$ \\
\hline & E2 & $77.92 * * \pm 0.51$ & $27.37^{* *} \pm 0.72$ & $2.97 \pm 0.07$ & $2.41^{* *} \pm 0.09$ & $0.41^{* *} \pm 0.01$ & $16.72 * * \pm 0.14$ & $0.27^{* *} \pm 0.01$ & - & - \\
\hline \multirow[t]{2}{*}{ [d] } & E1 & $14.89^{* *} \pm 0.46$ & $15.18^{* *} \pm 0.23$ & $-0.18^{* *} \pm 0.06$ & $-0.13 \pm 0.06$ & $-0.02 * \pm 0.01$ & $-0.14 * \pm 0.05$ & $-0.01 * \pm 0.00$ & - & - \\
\hline & E2 & $14.95^{* *} \pm 0.381$ & $14.85^{* *} \pm 0.26$ & $-0.46 \pm 0.08$ & $-0.44^{* *} \pm 0.09$ & $-0.08 * * \pm 0.01$ & $-0.59 * * \pm 0.14$ & $-0.05^{* *} \pm 0.01$ & - & - \\
\hline \multirow[t]{2}{*}[\mathrm{h}]{} & E1 & $15.04 * * \pm 0.84$ & $44.93^{* *} \pm 6.64$ & $0.34 * \pm 0.14$ & $0.45 \pm 0.14$ & $0.09 * * \pm 0.02$ & $0.32 * * \pm 0.11$ & $0.05^{* *} \pm 0.01$ & $-6.30 * * \pm 2.67$ & - \\
\hline & E2 & $14.86^{* *} \pm 0.83$ & $47.67 * * \pm 0.79$ & $1.74 \pm 0.12$ & $1.90 \pm 0.11$ & $0.38 \pm 0.06$ & $2.57 * * \pm 0.44$ & $0.26^{* *} \pm 0.04$ & - & - \\
\hline \multirow[t]{2}{*}{ [i] } & E1 & $-12.70 * *_{ \pm 0.95}$ & $16.63^{* *} \pm 3.07$ & $0.24 \pm 0.33$ & $0.24 \pm 0.33$ & $0.04 \pm 0.06$ & $0.04 \pm 0.29$ & $0.03 \pm 0.04$ & $-3.00^{* *} \pm 1.02$ & $-1.02 \pm 0.57$ \\
\hline & $\mathrm{E} 2$ & $-12.70 * *_{ \pm} 0.95$ & $16.63^{* *} \pm 2.17$ & $-0.28 \pm 0.32$ & $-0.28 \pm 0.32$ & $-0.05 \pm 0.06$ & $-0.24 \pm 0.29$ & $-0.02 \pm 0.04$ & - & - \\
\hline \multirow[t]{2}{*}{ [j] } & E1 & $-4.417 \pm 0.63$ & $-2.17 \pm 0.76$ & $-0.08 \pm 0.14$ & $-0.15 \pm 0.13$ & $-0.03 \pm 0.02$ & $-0.27 \pm 0.14$ & $-0.02 \pm 0.01$ & - & $1.03 * * \pm 0.22$ \\
\hline & $\mathrm{E} 2$ & $-5.03^{* *} \pm 0.53$ & $-1.87 * \pm 0.77$ & $0.50^{* *} \pm 0.13$ & $0.50^{* *} \pm 0.13$ & $0.09^{* *} \pm 0.02$ & $0.46^{* *} \pm 0.17$ & $0.07 * * \pm 0.01$ & - & - \\
\hline \multirow[t]{2}{*}[1]{} & E1 & $8.13^{* *} \pm 2.03$ & $-22.60 * * \pm 4.01$ & $-0.49 \pm 0.58$ & $-0.66 \pm 0.57$ & $-0.13 \pm 0.11$ & $-0.21 \pm 0.54$ & $-0.09 \pm 0.07$ & $3.79 * \pm 1.79$ & $-1.42 \pm 0.98$ \\
\hline & E2 & $5.70 * \pm 1.91$ & $-25.00 * \pm 3.38$ & $0.15 \pm 0.52$ & $-0.46 \pm 0.52$ & $-0.09 \pm 0.10$ & $-0.96 \pm 0.56$ & $-0.08 \pm 0.06$ & - & - \\
\hline \multirow[t]{2}{*}{$x^{2}$} & E1 & $16.04^{* *}$ & $8.10^{* *}$ & 1.06 & 3.04 & 3.10 & 5.96 & 3.24 & 5.72 & 1.88 \\
\hline & $\mathrm{E} 2$ & $8.86^{* *}$ & $5.79^{*}$ & 1.33 & 0.77 & 0.74 & 0.72 & 0.36 & - & - \\
\hline Type & E1 & $\mathrm{C}$ & $\mathrm{D}$ & - & - & - & - & - & - & - \\
\hline interaction & $\mathrm{E} 2$ & $\mathrm{C}$ & $\mathrm{D}$ & - & - & - & - & - & - & - \\
\hline \multicolumn{11}{|c|}{$* \mathrm{P}=0.05 ;$ and $* * \mathrm{P}=0.01$} \\
\hline
\end{tabular}


Table 6. Estimates of scaling tests and genic effects in linseed for cross TL-43 x Binwa

\begin{tabular}{|c|c|c|c|c|c|c|c|c|c|c|c|}
\hline Traits & Locations & $\begin{array}{l}50 \text { per cent } \\
\text { flowering }\end{array}$ & $\begin{array}{l}\text { Days to } \\
\text { maturity }\end{array}$ & $\begin{array}{c}\text { Primary } \\
\text { branches per } \\
\text { plant }\end{array}$ & $\begin{array}{c}\text { Secondary } \\
\text { branches per } \\
\text { plant }\end{array}$ & $\begin{array}{c}\text { Capsules per } \\
\text { plant }\end{array}$ & $\begin{array}{l}\text { Seeds per } \\
\text { capsule }\end{array}$ & $\begin{array}{l}\text { Biological } \\
\text { yield per } \\
\text { plant }\end{array}$ & $\begin{array}{l}\text { Seed yield } \\
\text { per plant }\end{array}$ & Harvest Index & $\begin{array}{c}1000 \text {-seed } \\
\text { weight }\end{array}$ \\
\hline \multirow[t]{2}{*}{$\mathrm{A}$} & E1 & $5.75^{* *} \pm 0.29$ & $3.34 * * \pm 0.30$ & $-1.72 * * \pm 0.27$ & $-0.75 * * \pm 0.21$ & $4.75^{* *} \pm 0.85$ & $0.18 \pm 0.51$ & $0.01 \pm 0.28$ & $-0.09 \pm-0.67$ & $3.10 \pm 2.35$ & $0.07 \pm 0.07$ \\
\hline & E2 & $1.33^{* *} \pm 0.38$ & $-1.67^{* *} \pm 0.42$ & $-2.46^{* * \pm 0} \pm .23$ & $-1.67 * * \pm 0.17$ & $17.92 * * \pm 1.34$ & $1.07^{* *} \pm 0.39$ & $-0.26 \pm 0.19$ & $0.05 \pm 0.10$ & $3.41 \pm 1.81$ & $0.07 \pm 0.06$ \\
\hline \multirow[t]{2}{*}{ B } & E1 & $-0.44 \pm 0.30$ & $33.86^{* *} \pm 0.33$ & $-0.65 \pm 0.37$ & $0.55^{*} \pm 0.22$ & $-7.54 * *_{ \pm 0.74}$ & $-0.20 \pm 0.37$ & $-0.34 \pm 0.31$ & $0.19 \pm 1.54$ & $5.79 * * \pm 2.01$ & $-0.10 \pm 0.08$ \\
\hline & E2 & $-0.33 \pm 0.31$ & $33.82 * * \pm 0.34$ & $-2.14 * * \pm 0.35$ & $-1.31 * * \pm 0.23$ & $19.32 * * \pm 1.48$ & $0.47 \pm 0.31$ & $0.00 \pm 0.26$ & $0.49 * * \pm 0.10$ & $9.87 * * \pm 2.02$ & $0.54 * * \pm 0.07$ \\
\hline \multirow[t]{2}{*}{$\mathrm{C}$} & E1 & $-0.46 \pm 0.49$ & $36.50^{* *} \pm 0.57$ & $-4.46 * * \pm 0.55$ & $-3.13 * * \pm 0.29$ & $55.76^{* *} \pm 1.51$ & $0.40 \pm 0.91$ & $0.86 \pm 0.43$ & $0.03 \pm 0.18$ & $2.33 \pm 3.44$ & $-0.84 * * \pm 0.14$ \\
\hline & E2 & $-0.46 \pm 0.49$ & $36.50^{* *} \pm 0.57$ & $-5.40 * * \pm 0.49$ & $-4.03 * * \pm 0.28$ & $53.96^{* *} \pm 1.23$ & $1.90^{* * \pm 0.62}$ & $0.53 \pm 0.36$ & $0.57^{* *} \pm 0.15$ & $8.09 * * \pm 2.95$ & $0.79 * * \pm 0.13$ \\
\hline \multirow[t]{2}{*}{$\mathrm{D}$} & E1 & $-2.88^{* *} \pm 0.18$ & $-2.98 * * \pm 0.23$ & $-1.04 * * \pm 0.11$ & $-1.46 * * \pm 0.14$ & $26.48 * * \pm 0.75$ & $0.20 \pm 0.34$ & $0.59 * * \pm 0.18$ & $-0.03 \pm-0.33$ & $-3.28 * \pm 1.43$ & $-0.40^{* *} \pm 0.04$ \\
\hline & E2 & $0.73 * * \pm 0.22$ & $0.50 \pm 0.28$ & $0.39 * * \pm 0.12$ & $0.52 * * \pm 0.14$ & $8.36^{* *} \pm 1.06$ & $0.17 \pm 0.25$ & $0.40 * \pm 0.16$ & $0.01 \pm 0.07$ & $-2.59 \pm 1.46$ & $0.16^{* *} \pm 0.03$ \\
\hline \multirow[t]{2}{*}[\mathrm{m}]{} & E1 & $106.73^{* *} \pm 0.39$ & $183.08^{* *} \pm 0.14$ & $2.92 * * \pm 0.19$ & - & $-23.10 * * \pm 1.53$ & $7.63^{* *} \pm 0.06$ & $6.45^{* *} \pm 0.39$ & $1.68^{* *} \pm 0.04$ & $22.95^{* *} \pm 3.01$ & $6.51^{* * \pm 0.08}$ \\
\hline & E2 & $101.04 \pm 0.46$ & $168.08 \pm 0.14$ & $4.36^{* *} \pm 0.27$ & $1.63^{* *} \pm 0.28$ & $11.17^{* *} \pm 0.33$ & $7.34^{* *} \pm 0.11$ & $5.30^{* *} \pm 0.06$ & $1.46^{* *} \pm 0.04$ & $28.27^{* *} *_{ \pm 0} .68$ & $6.97^{* *} \pm 0.05$ \\
\hline \multirow[t]{2}{*}{ [d] } & E1 & - & $15.41 * * \pm 0.14$ & - & - & $-4.15^{* *} \pm 0.21$ & $-0.78 * * \pm 0.09$ & $-0.21 * \pm 0.09$ & $-0.20 * * \pm 0.04$ & $-4.05^{* *} \pm 0.69$ & $-0.19 * * \pm 0.01$ \\
\hline & E2 & - & $15.41 \pm 0.14$ & $-0.43^{* *} \pm 0.07$ & $-0.31 * * \pm 0.06$ & $-4.36^{* *} \pm 0.21$ & $-0.28 * * \pm 0.09$ & - & $0.08 * \pm 0.04$ & - & $0.21^{* *} \pm 0.01$ \\
\hline \multirow[t]{2}{*}{ [h] } & E1 & $22.44 * * \pm 1.0$ & $44.60 * * \pm 0.50$ & $1.12 * * \pm 0.33$ & $3.73 * * \pm 0.16$ & $99.72 * * \pm 3.68$ & - & $-2.35^{*} \pm 1.05$ & $0.22 * * \pm 0.08$ & $27.23 * *_{ \pm} 7.83$ & $1.78^{* *} \pm 0.21$ \\
\hline & E2 & $9.51 \pm 1.24$ & $48.20 \pm 0.53$ & $-3.60 * * \pm 0.72$ & $-1.55 * \pm 0.75$ & - & $2.23^{* *} \pm 0.46$ & $0.15 \pm 0.12$ & $1.10^{* *} \pm 0.14$ & $19.13^{* *} *_{ \pm 2} .63$ & $0.41^{* *} \pm 0.09$ \\
\hline \multirow[t]{2}{*}{ [i] } & E1 & $5.77^{* *} \pm 0.36$ & $5.97 * * \pm 0.47$ & $2.08 * * \pm 0.23$ & $2.93^{* *} \pm 0.28$ & $52.97 * * \pm 1.51$ & $-0.41 \pm 0.68$ & $-1.19^{* *} \pm 0.37$ & $0.06 \pm 0.18$ & $6.56^{*} \pm 2.87$ & $0.81^{* *} \pm 0.08$ \\
\hline & E2 & $1.46^{* * \pm 0.43}$ & $1.00 \pm 0.56$ & $0.78^{* *} \pm 0.25$ & $1.04 * * \pm 0.28$ & $16.72 * * \pm 2.12$ & $-0.34 \pm 0.51$ & $-0.80 * \pm 0.32$ & $-0.02 \pm 0.14$ & $5.19 \pm 2.92$ & $0.32 * * \pm 0.07$ \\
\hline \multirow[t]{2}{*}{ [j] } & E1 & $3.10^{* *} \pm 0.18$ & $18.60^{* *} \pm 0.19$ & $-0.53^{* *} \pm 0.18$ & $-0.65^{* *} \pm 0.14$ & $6.15^{* *} \pm 0.50$ & $0.19 \pm 0.21$ & $0.17 \pm 0.18$ & $-0.14 \pm 0.08$ & $-1.34 \pm 1.38$ & $0.09 * \pm 0.04$ \\
\hline & E2 & $0.83^{* *} \pm 0.22$ & $16.07 * * \pm 0.25$ & $-0.16 \pm 0.17$ & $-0.18 \pm 0.13$ & $0.70 \pm 0.95$ & $0.30 \pm 0.20$ & $-0.13 \pm 0.14$ & $-0.22 * * \pm 0.06$ & $-3.22 * \pm 1.24$ & $0.31^{* *} \pm 0.02$ \\
\hline \multirow[t]{2}{*}{ [1] } & E1 & $-11.08^{* *} \pm 0.68$ & $24.54 * * \pm 0.78$ & $0.28 \pm 0.62$ & $-2.73^{* *} \pm 0.54$ & $-50.18^{* *} \pm 2.32$ & $0.42 \pm 1.17$ & $1.52 * \pm 0.72$ & $-0.16 \pm 0.32$ & $-15.45^{* *} \pm 5.26$ & $-0.77^{* *} \pm 0.17$ \\
\hline & E2 & $-2.46^{* *} \pm 0.84$ & $34.50 * * \pm 1.00$ & $3.82 * * \pm 0.61$ & $1.94 * * \pm 0.52$ & $20.52 * * \pm 3.92$ & $-1.21 \pm 0.90$ & $1.07 \pm 0.58$ & $-0.53 * \pm 0.26$ & $-18.47^{* *} \pm 5.05$ & $0.15 \pm 0.15$ \\
\hline \multirow[t]{2}{*}{$\chi^{2}$} & E1 & 1.31 & $158.02 * *$ & 3.17 & 4.81 & $149.40^{* *}$ & 2.90 & 0.87 & 3.41 & 0.94 & $5.24^{*}$ \\
\hline & E2 & 1.31 & 3.10 & 0.84 & 1.87 & 0.57 & 3.04 & $4.00^{*}$ & 0.02 & 4.23 & 0.90 \\
\hline \multirow{2}{*}{$\begin{array}{c}\text { Type of } \\
\text { interaction }\end{array}$} & E1 & - & C & - & - & $\mathrm{D}$ & - & - & - & - & D \\
\hline & E2 & - & - & - & - & - & - & $\mathrm{C}$ & - & - & - \\
\hline
\end{tabular}

Table 7. Estimates of scaling tests and genic effects in linseed for cross TL-43 x Binwa

\begin{tabular}{|c|c|c|c|c|c|c|c|c|c|c|}
\hline $\begin{array}{l}\text { Traits } \\
\text { Parameters }\end{array}$ & Locations & Plant height & $\begin{array}{c}\text { Technical } \\
\text { height }\end{array}$ & Straw weight & $\begin{array}{c}\text { Retted straw } \\
\text { weight }\end{array}$ & Total fibre & Percent fibre & Line fibre & Oil content & $\begin{array}{l}\text { Protein } \\
\text { content }\end{array}$ \\
\hline \multirow[t]{2}{*}{ A } & E1 & $19.86^{* *} \pm 1.12$ & $19.37 * * \pm 0.75$ & $0.00 \pm 0.24$ & $0.17 \pm 0.23$ & $0.03 \pm 0.04$ & $0.07 \pm 0.20$ & $0.02 \pm 0.03$ & $-4.50 * * \pm 0.72$ & $0.43 \pm .0 .39$ \\
\hline & E2 & $-34.56 * * \pm 1.39$ & $-26.45^{* *} \pm 0.97$ & $-0.02 \pm 0.17$ & $0.14 \pm 0.17$ & $0.02 \pm 0.03$ & $-0.00 \pm 0.14$ & $0.02 \pm 0.02$ & - & - \\
\hline \multirow[t]{2}{*}{ B } & E1 & $1.70 \pm 1.44$ & $2.18^{* *} \pm 0.79$ & $-0.45 \pm 0.26$ & $-0.27 \pm 0.25$ & $-0.05 \pm 0.05$ & $-0.38 \pm 0.22$ & $-0.03 \pm 0.03$ & $-12.19 * * \pm 0.69$ & $-6.54 * * \pm 0.35$ \\
\hline & E2 & $-7.21 * * \pm 1.71$ & $-1.54 \pm 1.00$ & $-0.20 \pm 0.24$ & $0.10 \pm 0.24$ & $0.02 \pm 0.04$ & $0.05 \pm 0.22$ & $0.01 \pm 0.03$ & - & - \\
\hline \multirow[t]{2}{*}{$\mathrm{C}$} & E1 & $54.33^{* *} \pm 2.92$ & $35.53 * * \pm 1.78$ & $0.69 \pm 0.38$ & $1.03 * * \pm 0.37$ & $0.20 * *_{ \pm 0} .07$ & $0.88 * * \pm 0.30$ & $0.13 * * \pm 0.05$ & $-1.09 \pm 1.27$ & $-2.97 * * \pm 0.65$ \\
\hline & E2 & $-51.30 * * \pm 2.42$ & $-33.86^{* *} \pm 1.56$ & $0.54 \pm 0.33$ & $1.02 * * \pm 0.34$ & $0.20 * * \pm 0.06$ & $0.78^{* *} \pm 0.27$ & $0.13 * * \pm 0.04$ & - & - \\
\hline \multirow[t]{2}{*}{ D } & E1 & $18.08^{* *} \pm 1.30$ & $-9.17 * * \pm 0.81$ & $0.56^{* *} \pm 0.16$ & $0.56^{* *} \pm 0.16$ & $0.11 * *_{ \pm} 0.03$ & $0.59 * * \pm 0.13$ & $0.07 * * \pm 0.02$ & $7.80 * * \pm 0.52$ & $1.57 * * \pm 0.26$ \\
\hline & E2 & $-4.75 * * \pm 1.49$ & $-2.93 * * \pm 0.87$ & $0.38^{*} \pm 0.15$ & $0.38^{*} \pm 0.15$ & $0.07 * \pm 0.03$ & $0.36^{* *} \pm 0.12$ & $0.05 * \pm 0.02$ & - & - \\
\hline \multirow[t]{2}{*}[\mathrm{m}]{} & E1 & $39.19^{* *} \pm 1.26$ & $24.09 * * \pm 0.79$ & $4.43^{* *} \pm 0.34$ & $3.21 * * \pm 0.04$ & $0.57 * * \pm 0.00$ & $18.59 * * \pm 0.27$ & $0.38^{* *} \pm 0.00$ & $54.17 \pm 1.08$ & $23.24 \pm 0.54$ \\
\hline & E2 & $44.66^{* *} \pm 0.60$ & $41.63 * * \pm 0.28$ & $3.40 * * \pm 0.03$ & $3.22 * * \pm 0.04$ & $0.57 * * \pm 0.00$ & $17.74 * * \pm 0.03$ & $0.38^{* *} \pm 0.00$ & - & - \\
\hline \multirow[t]{2}{*}{ [d] } & E1 & $8.05^{* *} \pm 0.52$ & $10.13^{* *} \pm 0.26$ & - & - & - & - & - & - & - \\
\hline & E2 & $10.14 * * \pm 0.41$ & $10.96 * * \pm 0.28$ & - & - & - & $0.15^{*} \pm 0.06$ & - & & \\
\hline \multirow[t]{2}{*}[\mathrm{h}]{} & E1 & $23.70 * * \pm 1.77$ & $17.05 * * \pm 1.13$ & $-2.60 * *_{ \pm} 0.91$ & - & - & $-2.25^{* *} \pm 0.74$ & - & $-46.53 * * \pm 2.67$ & $-11.62 \pm 1.37$ \\
\hline & E2 & - & $-31.20 * * \pm 1.25$ & - & - & - & - & - & - & - \\
\hline [i] & E2 & $9.51 * * \pm 2.98$ & $5.86^{* *} \pm 1.75$ & $-0.77 * \pm 0.30$ & $-0.77 * \pm 0.30$ & $-0.15^{*} \pm 0.06$ & $-0.73^{* *} \pm 0.24$ & $-0.10 * \pm 0.04$ & - & - \\
\hline \multirow[t]{2}{*}{ [j] } & E1 & $-10.78^{* *} \pm 0.78$ & $-10.78^{* *} \pm 0.45$ & $0.23 \pm 0.16$ & $0.22 \pm 0.15$ & $0.04 \pm 0.03$ & $0.22 \pm 0.13$ & $0.03 \pm 0.02$ & $10.79 * * \pm 0.42$ & $3.48 * * \pm 0.21$ \\
\hline & E2 & $-13.67 * * \pm 1.08$ & $-12.45^{* *} \pm 0.65$ & $0.08 \pm 0.13$ & $0.01 \pm 0.13$ & $0.00 \pm 0.02$ & $-0.02 \pm 0.12$ & $0.00 \pm 0.01$ & - & - \\
\hline \multirow[t]{2}{*}{ [1] } & E1 & $-18.00^{* * *} \pm 3.69$ & $-1.156 \pm 2.31$ & $1.57^{*} \pm 0.62$ & $1.23^{*} \pm 0.61$ & $0.24 * \pm 0.12$ & $1.50^{* * \pm 0} \pm .51$ & $0.16^{*} \pm 0.08$ & $32.35^{* *} \pm 1.77$ & $9.25^{* * \pm 0.93}$ \\
\hline & E2 & $32.26^{* *} \pm 4.68$ & $22.13 * * \pm 2.83$ & $1.01 \pm 0.54$ & $0.53 \pm 0.54$ & $0.10 \pm 0.10$ & $0.68 \pm 0.45$ & $0.06 \pm 0.07$ & - & - \\
\hline \multirow[t]{2}{*}{$\chi^{2}$} & E1 & $23.72 * *$ & 0.25 & 2.39 & 8.75 & 8.78 & 3.72 & 8.58 & $28.72 * *$ & 0.353 \\
\hline & E2 & $9.66^{* *}$ & $11.20^{* *}$ & 7.25 & $37.4 * *$ & 5.64 & 4.27 & 5.59 & - & - \\
\hline Type of & E1 & D & - & - & - & - & - & - & D & D \\
\hline interaction & E2 & - & $\mathrm{D}$ & - & - & - & - & - & - & - \\
\hline${ }^{*} \mathrm{P}=0.05$; and & 0.01 & & & & & & & & & \\
\hline
\end{tabular}


All the components of gene action i.e., $\mathrm{d}, \mathrm{h}, \mathrm{i}, \mathrm{j}$ and $\mathrm{l}$ in cross Turkey $\mathrm{x}$ Binwa were found to be significant for days to maturity and plant height in E2 whereas in other crosses in both the locations and above cross in E1 the similar behaviour was not observed. In addition in the above cross significantly positive value of $\mathrm{d}$ and significantly negative value of [i] for the above traits was observed. This indicates negative alleles were dispersed in the parents for the inheritance of these traits which suggests that the selection should be delayed to later generation. Foster, Pooni \& Mackay (1998) observed that dominance was high for plant height. Singh and Singh (1979) reported that the non-additive effects were more important for the genetic control of plant height in flax but present findings were inconsistent with findings of Sood et al (2007). Out of the three crosses TL - $11 \mathrm{x}$ Him Alsi-2 is the best cross in both the locations. This cross invariably has given 2-3 times higher yield than the remaining crosses. Therefore, the results obtained with respect to the TL $-11 \times$ Him Alsi-2 for generation mean analysis suggested that both additive and dominance gene effects were important for most of the traits but dominance was predominant as compared to additive effects. Duplicate type of gene action was observed in the expression of days to 50 per cent flowering, seed yield per plant, plant height, technical height and fibre traits in E2 for the above cross which implies the use of biparental approach and selection to be deferred to later generation. Complimentary type of gene action was seen for biological yield per plant, seed yield per plant which implies the use of biparental approach and early generation selection. Duplicate type of gene action for few traits in cross TL-11 x Him Alsi- 2, suggests to go for biparental matings or few cycles of recurrent selection for the accumulation of favourable genes for seed yield and its components in linseed. Moreover, for cross TL-11 x Him Alsi-2 in E2, additive $\mathrm{x}$ additive (i), additive $\mathrm{x}$ dominance (j) and dominance $\mathrm{x}$ dominance (l) interaction effects with duplicate nature are contributing in the inheritance of these traits viz., days to 50 per cent flowering, seed yield per plant, plant height, technical height, straw weight, retted straw weight, total fibre, per cent fibre, line fibre and capsules per plant, 1000 seed weight, plant height, technical height, oil content indicating recurrent selection could be employed for genetic improvement of these traits. The method is helpful in breaking up undesirable linkages. It was also suggested by Rao and Singh (1984) that dominance x dominance type of interaction played a significant role in the expression of some quantitative characters.

From the present study, it can be concluded that both additive and dominance effects were important for most of the traits but dominance was predominant as compared to additive effects. Duplicate type of gene action was observed in the expression of days to 50 per cent flowering, seed yield per plant, plant height, technical height and fibre traits at E2 for TL-11 x Him Alsi-2, these observation implies the use of biparental approach and selection to be delayed to later generations. Complimentary type of gene action was seen for biological yield per plant, seed yield per plant for TL-11 x Him Alsi-2 at E1 location and implies the use of biparental approach and early generation selection to be followed. The significance of additive component will thus be useful for improvement through selection and greater magnitude of dominant gene effects compared to additive effects suggest that hybridization breeding may be more useful. Thus, the estimates of present study were useful for analyzing genetic architecture of important traits which suggests future breeding strategy for improvement of desired traits.

\section{References}

Anderson, V. L., \& Kempthorne, O. (1954). A model for the study of quantitative inheritance. Genetics, 39, 883-898.

Anonymous. (2006). Annual Progress Report, All India Co-ordinated Research Project on linseed. Project Co-ordinating linseed unit, CSAUA \& T, Campus, Kanpur.

AOAC. (1970). Official methods of Analysis of the Association of Official Analytical Chemists. $11^{\text {th }}$ Edn. Washington D. C.

Cavalli, L. L. (1952). An analysis of linkage in quantitative inheritance. Quantitative Inheritance, H.M.S.O., London, pp. 135-144.

Doshi, S. P., \& Gupta, K. C. (1991). SPAR-1 Software. Indian Agricultural Statistical Research, New Delhi, India.

Foster, R., Pooni, H. S., \& Mackay, I. J. (1998). Quantitative analysis of Linum usitatissimum crosses for dual-purpose traits. Journal of Agricultural Science, Cambridge, 131, 285-292.

Ganesh, S. K., \& Sakila, M. (1999). Generation mean analysis in sesame (Sessamum indicum L.) crosses. Sesame and Safflower News Letter No, 14, 8-14.

Gauraha, D., \& Rao, S. S. (2011). Studies on Gene Action for Yield and Yield Attributing Characters in linseed (Linum usitatissimum L.). Journal of Agricultural Sciences, 2(1), 44-48. 
Hayman, B. I. (1958). The separation of epistatic from additive and dominance variations in generation means. Heredity, 12, 371-390. http://dx.doi.org/10.1038/hdy.1958.36

Hayman, B. I., \& Mather, K. (1955). The description of genic interactions in continous variation. Biometrics, 11, 69-82.

Jinks, J. L. (1983). Biometrical genetics of heterosis. In Frankel, R. (ed.) Heterosis, Springer-Verlag, Berlin, pp. $1-46$.

Jinks, J. L., \& Jones, R. M. (1958). Estimation of the components of heterosis. Genetics, 43, 223-234.

Lay, C. L., \& Dybing, C. D. (1989). Linseed. pp. 416 - 430 In: Oil Crops of the World edited by G. Röbbelen, R. K. Downey and A. Ashri. McGraw-Hill, New York.

Mather, K., \& Jinks, J. L. (1982). Biometrical Genetics, Third edition, Chapman and Hall, London.

Patel, J. A., Gupta, Y. K., Patel, S. B., \& Patel, J. N. (1997). Combining ability analysis over environments in linseed. Madras Agricultural Journal, 84,188-191.

Rao, S. K., \& Singh, S. P. (1984). Genetic analysis of yield and its components in linseed. Indian Journal of Agricultural Sciences, 54, 894-900.

Singh, J., Singh, K., Prakash, O., \& Vajpeyi, M. (2006). Seed colour-note a diagnostic character for disease resistance in linseed ( Linum usitatissimum L.). Indian Phytopathology, 59, 180-182.

Singh, K. P., \& Singh, H. G. (1979). Combining ability analysis for quantitative traits in linseed. Indian Journal of Agricultural Sciences, 49, 573-578.

Sood, S., Kalia, N. R., Bhateria. S., \& Kumar, S. (2007). Detection of genetic components of variation for some biometrical traits in Linum usitatissimum L. in sub-mountain Himalayan region, Euphytica, 155(1-2), 107-115. http://dx.doi.org/10.1007/s10681-006-9309-y

Tak, G. M. (1996). Gene action in Linseed. Advances in Plant Sciences, 9, 99-102.

Tyson, H. (1989). Genetic control of seed weight in flax (Linum usitatissimum) and possible implications. Theoretical Applied Genetics, 77, 260-270. http://dx.doi.org/10.1007/BF00266196 